\title{
Development and Validation of a Nomogram for Patients with Nonmetastatic BCLC Stage C Hepatocellular Carcinoma after Stereotactic Body Radiotherapy
}

\author{
Wen-Yen Huanga, b Chiao-Ling Tsaic, d Jenny Y. Que ${ }^{e, f}$ \\ Cheng-Hsiang Lo ${ }^{a}$ Yu-Ju Lin ${ }^{b}$ Yang-Hong Dai ${ }^{a}$ Jen-Fu Yang ${ }^{a}$ \\ Po-Chien Shen ${ }^{a} \quad$ Mei-Hsuan Lee ${ }^{b}$ Jason Chia-Hsien Cheng ${ }^{c, d}, g$ \\ ${ }^{a}$ Department of Radiation Oncology, Tri-Service General Hospital, National Defense Medical \\ Center, Taipei, Taiwan; ' Institute of Clinical Medicine, National Yang-Ming University, Taipei, \\ Taiwan; ' ${ }^{\circ}$ Division of Radiation Oncology, Department of Oncology, National Taiwan University \\ Hospital, Taipei, Taiwan; d Graduate Institute of Clinical Medicine, National Taiwan University \\ College of Medicine, Taipei, Taiwan; ${ }^{e}$ Department of Radiation Oncology, Chi Mei Medical \\ Center, Tainan, Taiwan; ${ }^{f}$ Department of Hospital and Health Care Administration, Chia \\ Nan University of Pharmacy and Science, Tainan, Taiwan; ${ }^{9}$ Graduate Institute of Oncology, \\ National Taiwan University College of Medicine, Taipei, Taiwan
}

\section{Keywords}

Hepatocellular carcinoma $\cdot$ Stereotactic body radiotherapy $\cdot$ Nomogram

\begin{abstract}
Background: Stereotactic body radiotherapy (SBRT) is an emerging treatment modality for hepatocellular carcinoma (HCC) with promising outcome. However, appropriate survival prediction models are scarce. This study aimed to develop a simple and clinically useful prognostic nomogram for patients with nondistant metastatic Barcelona Clinic Liver Cancer (BCLC) stage C HCC undergoing SBRT. Methods: The data were based on a prospective multi-institutional registry enrolling 246 patients with nondistant metastatic BCLC stage C HCC treated with SBRT between January 1, 2008 and December 31, 2016. They were randomly divided into two subsets: 164 into the development cohort and 82 into the validation cohort. We identified and included prognostic factors for survival to derive a nomogram in the development cohort. The predictability of the nomogram was evaluated in the validation cohort. The area under the receiver operating characteristic curve (AUROC) and the calibration plot were used to evaluate the performance of the nomogram. Results: The median survival was 13.5 months, with 1- and 2-year overall survival (OS) rates of 55.0 and $32.9 \%$, respectively. Number of tumors, largest tumor size, macrovascular invasion, Child-Turcotte-Pugh class, and biologically effective dose were significantly associated with OS $(p<0.05)$. These predictors were included to develop a nomogram with an AUROC of $0.77(0.73-0.87)$. The prediction model was well
\end{abstract}

Jason Chia-Hsien Cheng, MD, PhD

Division of Radiation Oncology, Department of Oncology

National Taiwan University Hospital, No. 7, Chung-Shan South Road

Zhongzheng District, Taipei 100 (Taiwan)

jasoncheng@ntu.edu.tw
Mei-Hsuan Lee, PhD

Institute of Clinical Medicine, National Yang-Ming University

Li-Nong Street, Section 2, Peitou

Taipei 112 (Taiwan)

meihlee@ntu.edu.tw or meihlee@ym.edu.tw 
calibrated in the validation cohort. The OS for patients who were divided by their risk scores differed significantly $(p<0.001)$. Conclusions: The nomogram we generated had discriminatory and satisfactory predictability for OS among nonmetastatic BCLC stage C HCC patients treated with SBRT. It demands further validations with cross-country data to confirm its worldwide usefulness.

(C) 2020 The Author(s)

Published by S. Karger AG, Basel

\section{Introduction}

Hepatocellular carcinoma (HCC), a complicated cancer, presents one of the most important challenges for oncologists. Although surveillance programs have been introduced for risk groups, HCC is often diagnosed at advanced stages when treatment selections are limited. The Barcelona Clinic Liver Cancer (BCLC) staging system predicts the prognosis and is linked to the suggested treatment modalities [1]. BCLC stage C HCC represents a large spectrum of disease with poor prognosis and constitutes the majority of patients. The treatment options for this stage are limited, with sorafenib monotherapy as the standard of care. However, a response rate of only $2-3.3 \%$ and a modest overall survival (OS) benefit of only 2-3 months by sorafenib raise the question about whether its use is appropriate in all BCLC stage $C$ patients [2-4].

Recently, several prospective studies have reported an encouraging outcome of stereotactic body radiotherapy (SBRT) for selected HCC patients with response rates of 40-70\% (complete and partial responses), 1-year local control rates of 75-95\%, and OS rates of $55-94 \%$ [5-10]. A randomized trial on the comparison between sorafenib alone and transcatheter arterial chemoembolization (TACE) followed by fractionated radiotherapy (RT) demonstrated superior response and survival by TACE plus RT and supported the role of RT in the multimodality treatments of patients with macrovascular invasion (MVI) [11]. However, patients with this stage vary tremendously in tumor burden, liver function, and performance status; this explains why there are large variations in treatment outcome. Besides, disease progression is frequently encountered when the selected treatment is not effective. Patients, if refractory in this situation, may have inadequate liver reserve to receive salvage treatment. Thus, accurate selection of treatment modalities is particularly crucial for this patient population, and a pragmatic and reliable nomogram for patients with BCLC stage C disease is particularly crucial to provide accurate outcome estimation before treatment, share decisionmaking evidence in patient-physician communication, and help select specific risk group of patients for future clinical trials.

In this study, we developed a simple and clinically useful nomogram for predicting OS in nondistant metastatic BCLC stage C HCC patients treated with SBRT. The quantitative effects of significant prognostic factors were also assessed and integrated into a prediction model with a continuum of outcome probabilities. Internal validation was performed to evaluate the reliability of the established model.

\section{Subjects and Methods}

Study Population and Data Collection

The patients in this study were collected in three medical centers in Taiwan (National Taiwan University Hospital, Tri-Service General Hospital, and Chi Mei Medical Center) using a standardized protocol. HCC patients who underwent SBRT for liver tumor(s) from January 1, 2008 to December 31, 2016 were enrolled.

The eligibility criteria included (1) patients diagnosed with HCC by dynamic imaging criteria and/or biopsy, (2) nondistant metastatic BCLC stage C, based on the presence of symptomatic tumors (e.g., Eastern 
Cooperative Oncology Group [ECOG] performance status score of 1-2) or vascular invasion/lymph node metastasis, (3) Child-Turcotte-Pugh (CTP) class A-B liver function, and (4) SBRT as one of the main local treatments. Most patients were discussed in a multidisciplinary team and RT was suggested to be beneficial for local control and/or survival. The reasons that patients were treated with SBRT included (1) unsuitable for TACE or refractory to TACE, (2) an alternative to sorafenib, (3) combination with TACE, and (4) progression after sorafenib. The treated target(s) by RT were intrahepatic and macrovascular tumors. We included all tumors if possible. However, for those who had poor liver function or limited normal liver volume, we adjusted radiation field and dose to prevent radiation liver injury. Our general principle was to deliver a radiation dose of $40 \mathrm{~Gy}$ in 5 fractions (biologically effective dose [BED] $=72 \mathrm{~Gy}$, with an alpha/beta ratio of 10). However, we allowed radiation dose adjustment according to the severity of liver cirrhosis, normal liver volume, or distance from luminal gastrointestinal tissue. For some small tumors at the liver margin, dose escalation to a BED of $100 \mathrm{~Gy}$ or higher was allowed. The dose constraints of organ at risk were previously described [12]. The use of sorafenib from the Taiwan National Health Insurance System was reimbursed to patients with good performance status, CTP class A, and extrahepatic metastasis or MVI. Patients eligible for using sorafenib from the Taiwan National Health Insurance System were prescribed sorafenib. Patients ineligible according to the criteria had the choice of paying the drug by themselves.

The data source of this study was from a prospective clinical data collection. The clinical information of the HCC patients was prospectively collected with a structured medical form, including age, sex, etiology (HBV, HCV, and non-B, non-C), ECOG performance status, prior use of sorafenib, combined use of sorafenib with SBRT, number of tumors, tumor size, alpha-fetoprotein (AFP) level, presence of MVI, CTP class, N stage, $\mathrm{M}$ stage, and BED. Patients' survival time was calculated from the end of SBRT to the date of death or last contact.

\section{Study Endpoints}

The primary aim of this study was to derive a prognostic OS model based on patient and tumor characteristics. OS was considered the most unbiased and reliable endpoint in most studies of advanced HCC patients. The cause of death of patients other than hepatic origin was assumed to be low [13].

\section{Statistical Analyses}

Overall, 246 HCC patients were randomly divided into two groups: 164 and 82 patients were assigned to the development and to the validation cohort, respectively. The differences in baseline characteristics of the patients in these two cohorts were examined by $\chi^{2}$ tests and Student's $t$ tests for categorical and continuous variables, respectively. Cox proportional hazard models were used to estimate hazard ratios with $95 \%$ confidence intervals (CIs) to evaluate the associations between predictors and 1-year survival. Predictors with statistically significant hazard ratios were included in the risk prediction models. The Cox proportional hazards regression coefficients for each predictor included in the risk model were converted into integer scores by rounding the quotients of dividing each regression coefficient by a single constant. The constant selected was the regression coefficient for the 5-year decrease in age, allowing the integer risk score for a 5 -year decrease in age to be 1 . The sum of scores was derived by adding the scores assigned to each predictor included in the model. The predicted risk of death within 1 year among the patients was estimated according to the following equation:

$$
\left.\left.\left.1-S_{0}(t)^{\exp \left\{\left[\beta_{\text {age }}(100-\text { base age })+\beta_{\text {age } 5 y}(\text { sum score })\right.\right.}\right)\right]-\sum_{i=1}^{p} \beta_{i} \bar{X}_{i}\right\}
$$

where $S_{0}(t)$ is the estimate of the average survival at 1 year, $\beta_{\text {age }}$ is the regression coefficient of age; $\beta_{\text {age }} y_{y}$ is the regression coefficient for each 5 -year decrement in age, $\beta_{i}$ is the regression coefficient for the ith variable, and denotes the mean value or proportion of the ith variable.

The receiver operating characteristic (ROC) curve was derived and the area under the ROC curve (AUROC) was calculated. The ROC curve for the prediction of death within 1 year was derived and the AUROC was used to assess the predictive accuracy of the risk model. Calibration was assessed by plotting the observed risk versus the model-predicted risk of death among the patients. The groups without 1-year death were combined with the next group. To evaluate the discriminatory ability of the prediction model, patients were classified into three groups by their sum of risk scores; the 25th and 75th percentiles of the sum of risk scores were used as cutoff values. The survivals of risk groups were estimated by Kaplan-Meier methods, and the differences were tested by the log-rank test. $p<0.05$ was considered statistically significant for all tests. All statistical analyses were performed with SAS version 9.4 (SAS Institute, Cary, NC, USA).

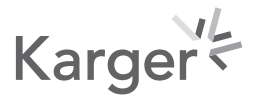


Huang et al.: Nomogram for Hepatocellular Carcinoma Treated with Stereotactic Body Radiotherapy

Table 1. Baseline characteristics of the patients in the development and validation cohorts

\begin{tabular}{|c|c|c|c|c|}
\hline \multirow[t]{2}{*}{ Characteristics } & \multicolumn{4}{|l|}{ Participants, $n(\%)$} \\
\hline & $\begin{array}{l}\text { total } \\
(N=246)\end{array}$ & $\begin{array}{l}\text { development } \\
(n=164)\end{array}$ & $\begin{array}{l}\text { validation } \\
(n=82)\end{array}$ & $p$ value \\
\hline \multicolumn{4}{|l|}{ Age } & 0.253 \\
\hline$<75$ years & $184(74.7)$ & $119(72.5)$ & $65(79.3)$ & \\
\hline$\geq 75$ years & $62(25.3)$ & $45(27.5)$ & $17(20.7)$ & \\
\hline \multicolumn{4}{|l|}{ Sex } & 0.118 \\
\hline Female & $63(25.6)$ & $38(23.1)$ & $25(30.5)$ & \\
\hline Male & $183(74.4)$ & $126(76.9)$ & $57(69.5)$ & \\
\hline \multicolumn{4}{|l|}{ Viral hepatitis } & 0.343 \\
\hline Non-B, non-C & 27 (10.9) & $15(9.1)$ & $12(14.6)$ & \\
\hline $\mathrm{HBV}$ & $135(54.9)$ & $90(54.9)$ & 45 (54.9) & \\
\hline $\mathrm{HCV}$ & $94(38.2)$ & $64(39.0)$ & $30(36.6)$ & \\
\hline HBV and HCV & $10(4.1)$ & $5(3.0)$ & $5(6.1)$ & \\
\hline \multicolumn{4}{|l|}{ ECOG performance status } & 0.712 \\
\hline 0 & $65(26.4)$ & $46(28.0)$ & $19(23.2)$ & \\
\hline 1 & $150(61.0)$ & $98(59.8)$ & $52(63.4)$ & \\
\hline 2 & $31(12.6)$ & $20(12.2)$ & $11(13.4)$ & \\
\hline \multicolumn{4}{|l|}{ Prior surgery } & 0.222 \\
\hline No & 206 (83.7) & 134 (81.7) & $72(87.8)$ & \\
\hline Yes & $40(16.3)$ & 30 (18.3) & $10(12.2)$ & \\
\hline \multicolumn{4}{|l|}{ Prior TACE } & 0.471 \\
\hline No & $125(50.8)$ & $86(52.4)$ & $39(47.6)$ & \\
\hline Yes & $121(49.2)$ & $78(47.6)$ & $43(52.4)$ & \\
\hline \multicolumn{4}{|l|}{ Prior use of sorafenib to SBRT } & 0.859 \\
\hline No & $229(93.1)$ & $153(93.3)$ & $76(92.7)$ & \\
\hline Yes & $17(6.9)$ & $11(6.7)$ & $6(7.3)$ & \\
\hline \multicolumn{4}{|c|}{ Combined use of sorafenib with SBRT } & 0.541 \\
\hline No & $206(83.7)$ & $139(84.8)$ & $67(81.7)$ & \\
\hline Yes & $40(16.3)$ & $25(15.2)$ & $15(18.3)$ & \\
\hline \multicolumn{4}{|l|}{ Combined TACE } & 0.215 \\
\hline No & $216(87.8)$ & $141(86.0)$ & $75(91.5)$ & \\
\hline Yes & $30(12.2)$ & $23(14.0)$ & $7(8.5)$ & \\
\hline \multicolumn{4}{|l|}{ Number of tumors } & 0.313 \\
\hline$\leq 3$ & $209(85.0)$ & $142(86.6)$ & 67 (81.7) & \\
\hline & $37(15.0)$ & $22(13.4)$ & $15(18.3)$ & \\
\hline \multicolumn{4}{|l|}{ Largest tumor size } & 0.203 \\
\hline Median/mean/IQR, cm & $5.5 / 6.5 / 3.5-8.9$ & $5.4 / 6.5 / 3.5-8.8$ & $6.4 / 6.5 / 3.3-9.8$ & \\
\hline$\leq 5 \mathrm{~cm}$ & $107(43.5)$ & $76(46.3)$ & $31(37.8)$ & \\
\hline$>5 \mathrm{~cm}$ & $139(56.5)$ & $88(53.7)$ & $51(62.2)$ & \\
\hline \multicolumn{4}{|l|}{ Pre-SBRT AFP } & 0.802 \\
\hline$\leq 20 \mathrm{ng} / \mathrm{mL}$ & $66(26.8)$ & $45(27.4)$ & $21(25.6)$ & \\
\hline \multirow{2}{*}{\multicolumn{4}{|c|}{$\underset{\text { MVI }}{>20 \mathrm{ng} / \mathrm{mL}}$}} & \\
\hline & & & & 0.302 \\
\hline No & $122(49.6)$ & $78(47.6)$ & $44(53.7)$ & \\
\hline Yes & $124(50.4)$ & $86(52.4)$ & $38(46.3)$ & \\
\hline \multicolumn{4}{|l|}{ CTP class } & 0.904 \\
\hline A & $205(83.3)$ & $137(83.5)$ & $68(82.9)$ & \\
\hline \multirow{2}{*}{\multicolumn{4}{|c|}{$\begin{array}{c}\text { B } \\
\text { N stage }\end{array}$}} & \\
\hline & & & & 0.215 \\
\hline 0 & $216(87.8)$ & $147(90.0)$ & $69(84.1)$ & \\
\hline 1 & $30(12.2)$ & $17(10.0)$ & $13(15.9)$ & \\
\hline
\end{tabular}

AFP, alpha-fetoprotein; CTP, Child-Turcotte-Pugh; ECOG, Eastern Cooperative Oncology Group; IQR, interquartile range; MVI, macrovascular invasion; SBRT, stereotactic body radiotherapy; TACE, transcatheter arterial chemoembolization. 


\section{Liver Cancer}

Table 2. Multivariate analysis of OS

\begin{tabular}{l|l}
\hline Liver Cancer 2020;9:326-337 \\
\hline DOI: 10.1159/000505693 & $\begin{array}{l}\text { @ 2020 The Author(s). Published by S. Karger AG, Basel } \\
\text { www.karger.com/lic }\end{array}$ \\
\hline
\end{tabular}

Huang et al.: Nomogram for Hepatocellular Carcinoma Treated with Stereotactic Body Radiotherapy

\begin{tabular}{lll}
\hline Characteristics & $\begin{array}{l}\text { Crude HR of death } \\
(95 \% \mathrm{CI})\end{array}$ & $p$ value \\
\hline $\begin{array}{l}\text { Number of tumors } \\
\quad \leq 3\end{array}$ & 1.00 & \\
$\quad>3$ & $2.31(1.08-2.39)$ & 0.003 \\
$\begin{array}{l}\text { Largest tumor size } \\
\quad \leq 5 \mathrm{~cm}\end{array}$ & \\
$\quad>5 \mathrm{~cm}$ & 1.00 & \\
MVI & $1.61(1.33-4.00)$ & 0.018 \\
$\quad$ No & 1.00 & \\
$\quad$ Yes & $2.55(1.72-3.77)$ & $<0.001$ \\
CTP class & & \\
$\quad \mathrm{A}$ & 1.00 & \\
$\quad \mathrm{~B}$ & $2.11(1.33-3.35)$ & 0.002 \\
BED (10-Gy increase) & $0.99(0.98-0.99)$ & 0.046 \\
\hline
\end{tabular}

BED, biologically effective dose; CI, confidence interval; CTP, ChildTurcotte-Pugh; HR, hazard ratio; MVI, macrovascular invasion; OS, overall survival.

\section{Results}

\section{Patterns of Progression and Survival Outcomes of All Patients}

The baseline characteristics of the patients are shown in Table 1. The SBRT dose prescribed ranged from 25 to 60 Gy in 2-6 fractions. The median BED was 86 Gy (interquartile range: $72-90 \mathrm{~Gy}$ ). The median follow-up time was 25.8 months for patients alive. Among the 246 patients, 176 deaths occurred after 4,729 person-months of follow-up. The median OS was 13.5 months (95\% CI 10.8-16.2). The median progression-free survival was 6.1 months (95\% CI 5.1-7.1). The most common pattern of progression was out-field intrahepatic progression (140 patients, $56.9 \%$ ), followed by extrahepatic progression (51 patients, $20.7 \%$ ) and in-field progression (40 patients, 16.3\%). The 1- and 2-year in-field failure-free rates were 80.8 and $78.2 \%$, respectively.

\section{Prognostic Factors in the Development Cohort}

The predictors of OS in univariate analysis are listed in online supplementary Table 1 (for all online suppl. material, see www.karger.com/doi/10.1159/000505693). All significant factors in univariate analysis were included in multivariate analysis with Cox proportional hazards models. After adjustment of all predictors, those remaining significantly associated with OS in multivariate analysis were number of tumors, largest tumor size, MVI, CTP class, and BED $(p<0.05)$ (Table 2). The outcomes and prognostic factors of a subgroup of patients with MVI were analyzed separately (online suppl. Tables 2 and 3).

\section{Nomogram for OS}

A nomogram including the significant prognostic factors was developed (Table 3; Fig. 1a). The nomogram illustrated MVI as sharing the largest contribution to OS. Number of tumor, tumor size, CTP class, and BED showed a moderate impact on OS. Each category within these variables was assigned a score on the point scale. By summing up the total score, the projections shown in Figure 1b indicated the estimated 1-year mortality risk.

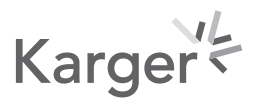


Huang et al:: Nomogram for Hepatocellular Carcinoma Treated with Stereotactic Body Radiotherapy

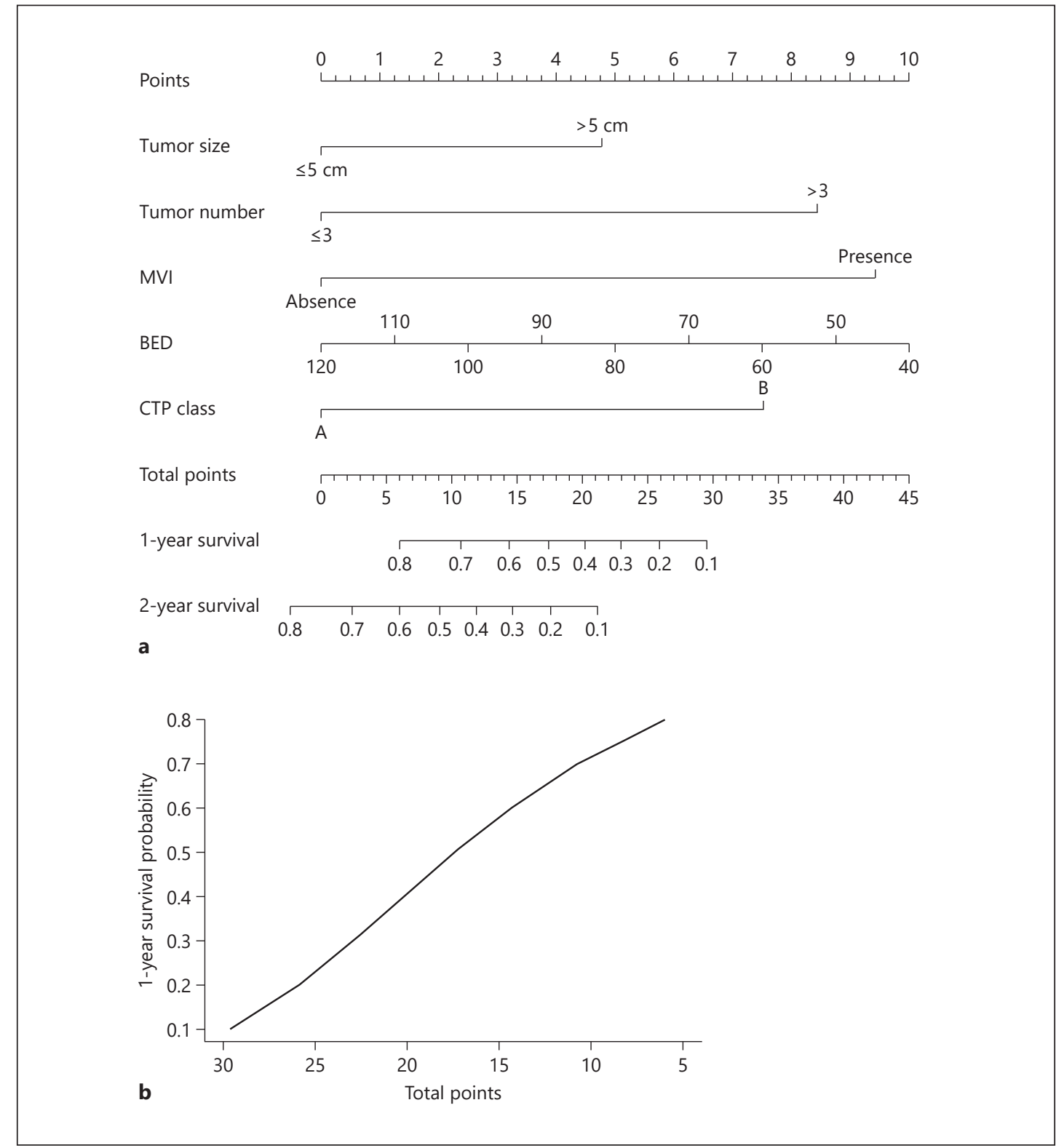

Fig. 1. Survival nomogram (a) and predicted risk of death within 1 year (b). The total points of each patient can be used to predict survival outcome. BED, biologically effective dose; CTP, Child-Turcotte-Pugh; MVI, macrovascular invasion.

\section{Predictive Accuracy and Calibration of the Nomogram}

The AUROC of the development cohort by the risk score was 0.77 (95\% CI 0.73-0.87) (Fig. 2). The calibration plots presented great agreement in both the development and the validation cohort between the nomogram prediction and actual observation (online suppl. Fig. 1).

\section{Discriminatory Ability of the Nomogram}

To test the discriminatory ability of the nomogram, we grouped the patients in both the development and the validation cohort into three subgroups according to their total risk score. They were divided by the 25 th and 75 th percentiles; the three groups differed distinctively (Fig. 3, $p<0.001$ ). 


\section{Liver Cancer}

Fig. 2. ROC curves and AUROCs for the prediction of death within 1 year among HCC patients after SBRT in the development and validation cohorts. AUROC, area under the receiver operating characteristic curve; HCC, hepatocellular carcinoma; ROC, receiver operating characteristic; SBRT, stereotactic body radiotherapy. (c) 2020 The Author(s). Published by S. Karger AG, Basel www.karger.com/lic

Huang et al.: Nomogram for Hepatocellular Carcinoma Treated with Stereotactic Body Radiotherapy

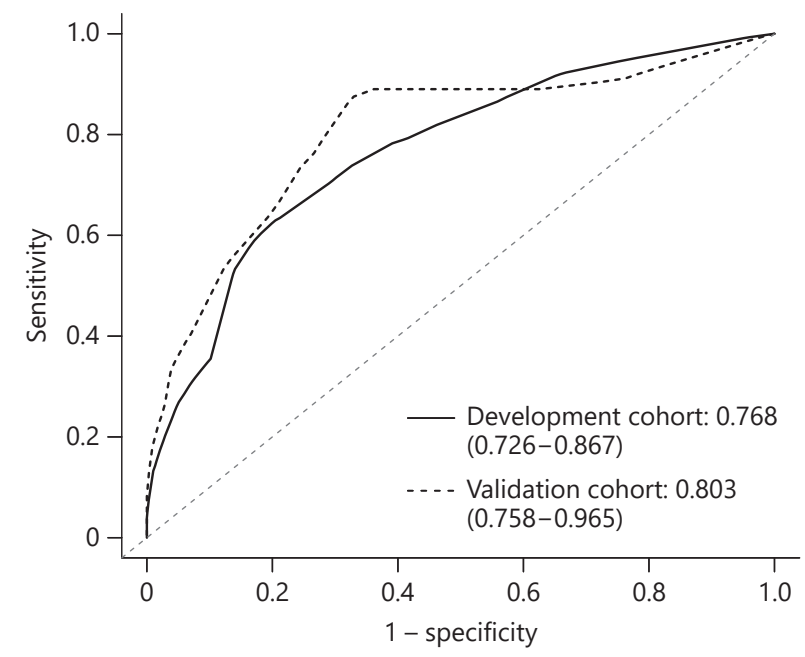

Table 3. Risk scores for survival prediction

\begin{tabular}{|c|c|c|c|c|}
\hline Risk predictors & $\begin{array}{l}\text { Multivariate HR of } \\
\text { death }(95 \% \mathrm{CI})\end{array}$ & $p$ value & $\begin{array}{l}\text { Beta } \\
\text { coefficient }\end{array}$ & $\begin{array}{l}\text { Risk } \\
\text { score }\end{array}$ \\
\hline \multicolumn{5}{|l|}{ Number of tumors } \\
\hline$\leq 3$ & 1.00 & & & 0 \\
\hline$>3$ & $2.31(1.08-2.39)$ & $<0.001$ & 0.838 & 8 \\
\hline \multicolumn{5}{|l|}{ Tumor size } \\
\hline$\leq 5 \mathrm{~cm}$ & 1.00 & & & 0 \\
\hline$>5 \mathrm{~cm}$ & $1.61(1.33-4.00)$ & 0.002 & 0.475 & 5 \\
\hline \multicolumn{5}{|l|}{ MVI } \\
\hline Absence & 1.00 & & & 0 \\
\hline Presence & $2.55(1.72-3.77)$ & $<0.001$ & 0.936 & 9 \\
\hline \multicolumn{5}{|l|}{ CTP class } \\
\hline $\mathrm{A}$ & 1.00 & & & 0 \\
\hline $\mathrm{B}$ & $2.11(1.33-3.35)$ & 0.009 & 0.747 & 8 \\
\hline BED, 10-Gy increase & $0.99(0.98-0.99)$ & 0.046 & 0.012 & \\
\hline$\leq 40$ & & & & 10 \\
\hline $41-50$ & & & & 9 \\
\hline $51-60$ & & & & 8 \\
\hline $61-70$ & & & & 7 \\
\hline $71-80$ & & & & 6 \\
\hline $81-90$ & & & & 4 \\
\hline $91-100$ & & & & 3 \\
\hline $101-110$ & & & & 2 \\
\hline $111-120$ & & & & 1 \\
\hline
\end{tabular}

BED, biologically effective dose; CI, confidence interval; CTP, Child-Turcotte-Pugh; HR, hazard ratio; MVI, macrovascular invasion. 

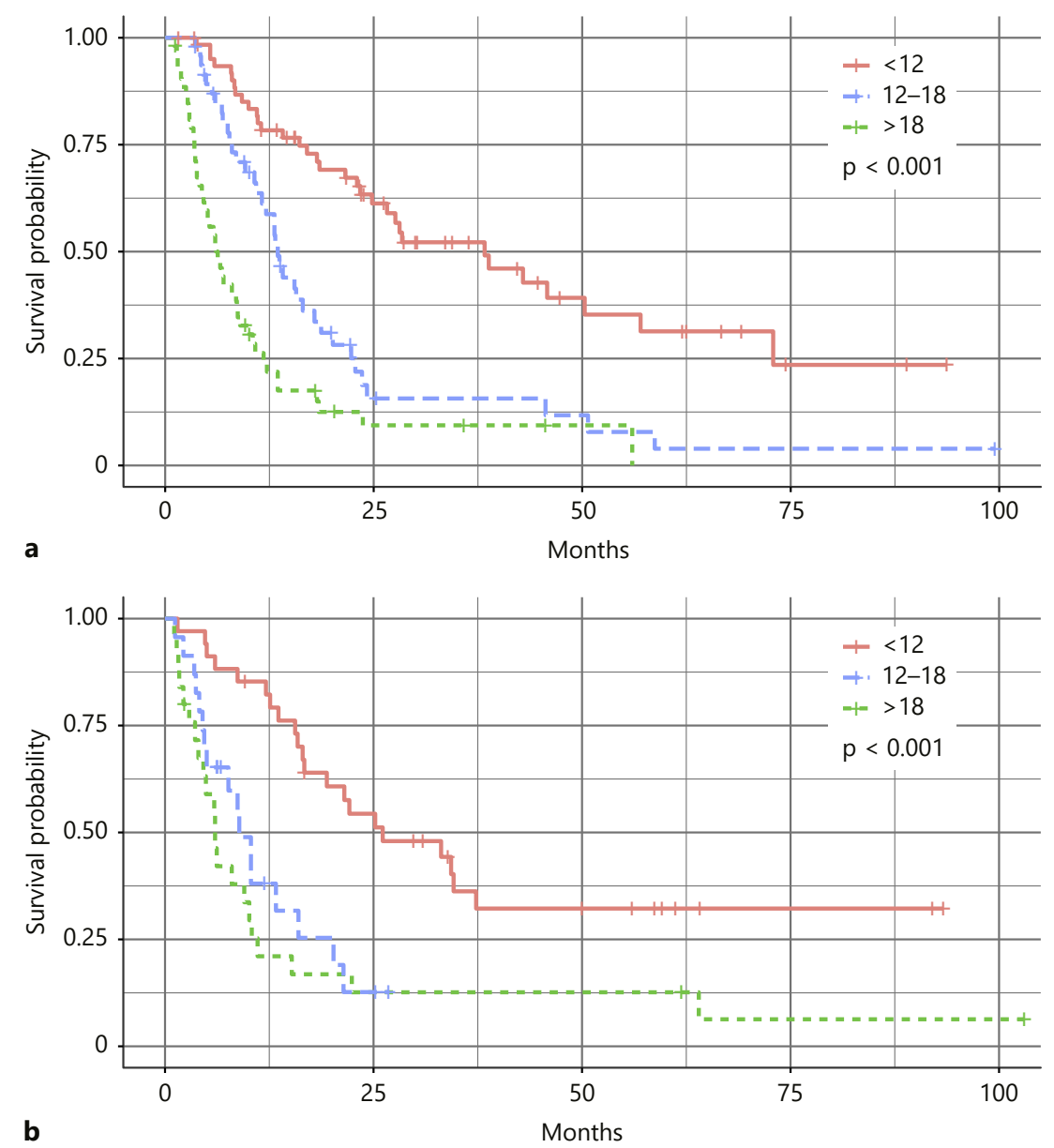

Fig. 3. Survival curves of patients with low (<12), medium (12-18), and high $(>18)$ risk scores: development cohort (a) and validation cohort (b). AUROC, area under the receiver operating characteristic curve; HCC, hepatocellular carcinoma; ROC, receiver operating characteristic; SBRT, stereotactic body radiotherapy.

\section{Discussion}

We developed and validated the nomogram to predict the 1-year survival probability of patients with BCLC stage C HCC treated with SBRT. The present nomogram, with high discrimination power, allows clinicians to skip complex calculations and simply use pretreatment clinical factors. The predicted survival highly correlated with the corresponding actual survival. Notably, this nomogram significantly integrated prognostic patient- and tumorrelated risk factors and provided a personalized estimation of OS. With the evolving evidence to show the advantage of combining SBRT with other liver-directed treatments in BCLC stage C patients [11], it is important to stratify patients for potential benefit of SBRT.

Compared to the median survival of 13.5 months in the present study, the two landmark trials on sorafenib (the SHARP trial and the Asia-Pacific trial) with unresectable and metastatic HCC patients had median survivals of 10.7 and 6.5 months, respectively [2, 3]. Most patients in these two trials had BCLC stage C disease (82 and 95\%, respectively). Notably, 
Huang et al.: Nomogram for Hepatocellular Carcinoma Treated with Stereotactic Body Radiotherapy

$18.2 \%$ of our patients had CTP class B compared with 5\% in the SHARP trial and $2.7 \%$ in the Asia-Pacific trial at enrollment. The median OS was 9.7 months for BCLC stage C patients in the SHARP trial [14]. Therefore, the survival of our patients with BCLC stage C disease treated with SBRT was not inferior to the standard care with sorafenib. Recently more targeted drugs, including lenvatinib, regorafenib, and cabozantinib, have shown their benefit in different subgroups of patients, but the survivals remain unsatisfactory [15-17]. Even with the promising response of immune checkpoint inhibitors, the impact on survival outcome is yet unsatisfactory $[18,19]$.

This study again demonstrates that advanced HCC patients with a high tumor burden show poor outcome after SBRT. Larger tumor number and size were associated with worse OS. There have been no generalized selection criteria of tumor volume, size, or number for HCC patients treated with SBRT. In this study, the largest tumor size or number of tumor were both significantly associated with OS in multivariate analysis. This reflects that intrahepatic disease burden was an independent factor of OS in HCC patients treated with SBRT.

MVI is not uncommon in patients with large tumors and highly associated with systemic dissemination and dismal outcome [20,21]. Compared to other criteria for BCLC stage C, MVI has an independent impact on survival. In our study, the median survival of patients with MVI was only 8.5 months, and MVI was a key component in the nomogram. Most patients in this study undergoing SBRT were unsuitable for or refractory to TACE and were different from patients with MVI treated with TACE followed by planned RT in a recent randomized trial with superior outcomes to sorafenib [11]. For the unique role of MVI, it may be essential to construct a nomogram specific for the subgroup of patients with MVI. Future investigations on this subgroup of patients based on different treatment combinations are needed.

Liver function was an independent prognosticator in our model. Numerous studies have clearly verified the importance of CTP class in patients with HCC, as essentially evidenced by nearly all staging systems [22].

Given the inherent nature of diverse HCC profiles, several complementary nomograms have been proposed and widely used for different treatment modalities. The Metroticket Investigator Study Group derived a prognostic model for patients undergoing transplantation [23]. Their nomogram for post-transplantation survival was based on largest size of tumor, number of tumors, and presence of microvascular invasion. Xu et al. [24] proposed a survival nomogram, with a predictive accuracy and discriminatory ability higher than seven commonly used staging systems, for patients with unresectable HCC treated with TACE. Vascular invasion, number of tumors, preserved tumor capsule, AFP level, aspartate aminotransferase level, and indocyanine green retention rate were independent prognosticators. Kao et al. [25] developed a nomogram to predict survival for early-stage HCC treated with radiofrequency ablation, consisting of age, prothrombin time international normalized ratio, AFP level, multiple tumors, and albumin-bilirubin grade. Besides, the predictive nomograms are available in surgical series [26, 27]. Variations in prognostic factors and patient inclusion criteria with their different impacts among these nomograms do exist for different liverdirected treatments.

In the present nomogram, the AUROCs for 1-year survival prediction were 0.77 and 0.80 for patients in the development and validation cohorts, respectively, indicating satisfactory prediction accuracy. The calibration plots for both cohorts also showed great agreement between survival distribution predicted by the nomogram and actual observation. The prognostic discrimination of the nomogram was featured by the model that was able to stratify patients into three prognostic subgroups with distinct outcomes. Altogether, these findings indicate that our nomogram is a reliable tool to predict survival for individual patients with BCLC stage $C$ undergoing SBRT. The nomogram aiming to integrate treatment modalities may

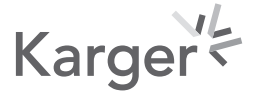


Huang et al.: Nomogram for Hepatocellular Carcinoma Treated with Stereotactic Body Radiotherapy

further optimize outcome prediction. Thus, to develop a nomogram specific for RT is essential. Except for the nomograms from HCC patients treated with fractionated RT for lymph node metastasis $[28,29]$, to our knowledge, our nomogram is the first linked to survival for stage $\mathrm{C}$ patients undergoing SBRT for primary liver tumors.

The current nomogram has some limitations. First, our study participants were all from Taiwan, which is an HBV endemic area with a high prevalence of HCC. The applicability of this nomogram to patients in other countries and/or with different etiologies is uncertain and needs to be validated. Second, the sample size was small. Although the use of SBRT has been more popular, the number of HCC patients treated with this modality is small. The present study is likely the largest to date that focused on SBRT to HCC. Third, various treatment modalities for disease progression after SBRT were not controlled in this study and could potentially have confounded the data interpretation. Lastly, we evaluated the presence of MVI, but not its extent. For our target population with BCLC stage C, MVI is only one of the criteria. Given the potential different-branch effect of MVI, the investigation on MVI extent is ongoing.

In conclusion, this simple-to-use nomogram derived from pretreatment clinical variables allows physicians to estimate the individualized survival of HCC patients treated with SBRT and can be used as a reference for decision-making by patients and/or physicians before treatment. It may be useful to select specific risk groups of patients for clinical trials. Our nomogram demands further validations with cross-country data to confirm its usefulness with the integration of SBRT into multidisciplinary treatments for patients with advanced HCC.

\section{Statement of Ethics}

The study received approval of our institutional review board (201407077RINA and 201609036RINC) with prospective clinical data collection. All protocols were carried out in accordance with the Helsinki Declaration of 1975 (revised in 1983). Consent was waived because this was an observational study.

\section{Disclosure Statement}

The authors have no conflicts of interest to declare.

\section{Funding Sources}

This study was supported in part by a study project grant from Chi Mei Medical Center, National Defense Medical Center (CMNDMC10805), Tri-Service General Hospital (TSGH-C107-035), and Liver Disease Prevention \& Treatment Research Foundation, Taiwan.

\section{Author Contributions}

Study concept and design: J.C.-H. Cheng, M.-H. Lee, W.-Y. Huang. Acquisition of data: W.-Y. Huang, C.-L. Tsai, J.Y. Que. Analysis and interpretation of data: C.-H. Lo, J.-F. Yang, P.-C. Shen, J.C.-H. Cheng. Drafting of the manuscript: W.-Y. Huang, C.-H. Lo, J.Y. Que. Critical revision of the manuscript for important intellectual content: all authors. Statistical analysis: M.-H. Lee, Y.-J. Lin, Y.-H. Dai. Study supervision: J.C.-H. Cheng.

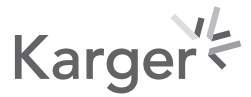




\begin{tabular}{|c|c|}
\hline Liver Cancer 2020;9:326-3 & \\
\hline DOI: 10.1159/000505693 & $\begin{array}{l}\text { (C) } 2020 \text { The Author(s). Published by S. Karger AG, Basel } \\
\text { www.karger.com/lic }\end{array}$ \\
\hline
\end{tabular}

Huang et al.: Nomogram for Hepatocellular Carcinoma Treated with Stereotactic Body Radiotherapy

\section{References}

1 Llovet JM, Brú C, Bruix J. Prognosis of hepatocellular carcinoma: the BCLC staging classification. Semin Liver Dis. 1999;19(3):329-38.

2 Llovet JM, Ricci S, Mazzaferro V, Hilgard P, Gane E, Blanc JF, et al.; SHARP Investigators Study Group. Sorafenib in advanced hepatocellular carcinoma. N Engl J Med. 2008 Jul;359(4):378-90.

3 Cheng AL, Kang YK, Chen Z, Tsao CJ, Qin S, Kim JS, et al. Efficacy and safety of sorafenib in patients in the AsiaPacific region with advanced hepatocellular carcinoma: a phase III randomised, double-blind, placebocontrolled trial. Lancet Oncol. 2009 Jan;10(1):25-34.

4 Bruix J, Cheng AL, Meinhardt G, Nakajima K, De Sanctis Y, Llovet J. Prognostic factors and predictors of sorafenib benefit in patients with hepatocellular carcinoma: analysis of two phase III studies. J Hepatol. 2017 Nov;67(5):999-1008.

5 Bujold A, Massey CA, Kim JJ, Brierley J, Cho C, Wong RK, et al. Sequential phase I and II trials of stereotactic body radiotherapy for locally advanced hepatocellular carcinoma. J Clin Oncol. 2013 May;31(13):1631-9.

6 Culleton S, Jiang H, Haddad CR, Kim J, Brierley J, Brade A, et al. Outcomes following definitive stereotactic body radiotherapy for patients with Child-Pugh B or C hepatocellular carcinoma. Radiother Oncol. 2014 Jun;111(3): 412-7.

7 Kang JK, Kim MS, Cho CK, Yang KM, Yoo HJ, Kim JH, et al. Stereotactic body radiation therapy for inoperable hepatocellular carcinoma as a local salvage treatment after incomplete transarterial chemoembolization. Cancer. 2012 Nov;118(21):5424-31.

8 Lasley FD, Mannina EM, Johnson CS, Perkins SM, Althouse S, Maluccio M, et al. Treatment variables related to liver toxicity in patients with hepatocellular carcinoma, Child-Pugh class A and B enrolled in a phase 1-2 trial of stereotactic body radiation therapy. Pract Radiat Oncol. 2015 Sep-0ct;5(5):e443-9.

9 Méndez Romero A, Wunderink W, Hussain SM, De Pooter JA, Heijmen BJ, Nowak PC, et al. Stereotactic body radiation therapy for primary and metastatic liver tumors: A single institution phase i-ii study. Acta Oncol. 2006;45(7):831-7.

10 Bettinger D, Pinato DJ, Schultheiss M, Sharma R, Rimassa L, Pressiani T, et al. Stereotactic Body Radiation Therapy as an Alternative Treatment for Patients with Hepatocellular Carcinoma Compared to Sorafenib: A Propensity Score Analysis. Liver Cancer. 2019 Jul;8(4):281-94.

11 Yoon SM, Ryoo BY, Lee SJ, Kim JH, Shin JH, An JH, et al. Efficacy and Safety of Transarterial Chemoembolization Plus External Beam Radiotherapy vs Sorafenib in Hepatocellular Carcinoma With Macroscopic Vascular Invasion: A Randomized Clinical Trial. JAMA Oncol. 2018 May;4(5):661-9.

12 Huang WY, Jen YM, Lee MS, Chang LP, Chen CM, Ko KH, et al. Stereotactic body radiation therapy in recurrent hepatocellular carcinoma. Int J Radiat Oncol Biol Phys. 2012 Oct;84(2):355-61.

13 Llovet JM, Di Bisceglie AM, Bruix J, Kramer BS, Lencioni R, Zhu AX, et al.; Panel of Experts in HCC-Design Clinical Trials. Design and endpoints of clinical trials in hepatocellular carcinoma. J Natl Cancer Inst. 2008 May; 100(10):698-711.

14 Bruix J, Raoul JL, Sherman M, Mazzaferro V, Bolondi L, Craxi A, et al. Efficacy and safety of sorafenib in patients with advanced hepatocellular carcinoma: subanalyses of a phase III trial. J Hepatol. 2012 Oct;57(4):821-9.

15 Abou-Alfa GK, Meyer T, Cheng AL, El-Khoueiry AB, Rimassa L, Ryoo BY, et al. Cabozantinib in Patients with Advanced and Progressing Hepatocellular Carcinoma. N Engl J Med. 2018 Jul;379(1):54-63.

16 Bruix J, Qin S, Merle P, Granito A, Huang YH, Bodoky G, et al.; RESORCE Investigators. Regorafenib for patients with hepatocellular carcinoma who progressed on sorafenib treatment (RESORCE): a randomised, doubleblind, placebo-controlled, phase 3 trial. Lancet. 2017 Jan;389(10064):56-66.

17 Kudo M, Finn RS, Qin S, Han KH, Ikeda K, Piscaglia F, et al. Lenvatinib versus sorafenib in first-line treatment of patients with unresectable hepatocellular carcinoma: a randomised phase 3 non-inferiority trial. Lancet. 2018 Mar;391(10126):1163-73.

18 El-Khoueiry AB, Sangro B, Yau T, Crocenzi TS, Kudo M, Hsu C, et al. Nivolumab in patients with advanced hepatocellular carcinoma (CheckMate 040): an open-label, non-comparative, phase 1/2 dose escalation and expansion trial. Lancet. 2017 Jun;389(10088):2492-502.

19 Zhu AX, Finn RS, Edeline J, Cattan S, Ogasawara S, Palmer D, et al.; KEYNOTE-224 investigators. Pembrolizumab in patients with advanced hepatocellular carcinoma previously treated with sorafenib (KEYNOTE224): a non-randomised, open-label phase 2 trial. Lancet Oncol. 2018 Jul;19(7):940-52.

20 Jonas S, Bechstein WO, Steinmüller T, Herrmann M, Radke C, Berg T, et al. Vascular invasion and histopathologic grading determine outcome after liver transplantation for hepatocellular carcinoma in cirrhosis. Hepatology. 2001 May;33(5):1080-6.

21 Cheng S, Chen M, Cai J, Sun J, Guo R, Bi X, et al. Chinese Expert Consensus on Multidisciplinary Diagnosis and Treatment of Hepatocellular Carcinoma with Portal Vein Tumor Thrombus (2018 Edition). Liver Cancer. 2020;9:28-40.

22 Guglielmi A, Ruzzenente A, Pachera S, Valdegamberi A, Sandri M, D’Onofrio M, et al. Comparison of seven staging systems in cirrhotic patients with hepatocellular carcinoma in a cohort of patients who underwent radiofrequency ablation with complete response. Am J Gastroenterol. 2008 Mar;103(3):597-604.

23 Mazzaferro V, Llovet JM, Miceli R, Bhoori S, Schiavo M, Mariani L, et al.; Metroticket Investigator Study Group. Predicting survival after liver transplantation in patients with hepatocellular carcinoma beyond the Milan criteria: a retrospective, exploratory analysis. Lancet Oncol. 2009 Jan;10(1):35-43. 
24 Xu L, Peng ZW, Chen MS, Shi M, Zhang YJ, Guo RP, et al. Prognostic nomogram for patients with unresectable hepatocellular carcinoma after transcatheter arterial chemoembolization. J Hepatol. 2015 Jul;63(1):122-30.

25 Kao WY, Su CW, Chiou YY, Chiu NC, Liu CA, Fang KC, et al. Hepatocellular Carcinoma: Nomograms Based on the Albumin-Bilirubin Grade to Assess the Outcomes of Radiofrequency Ablation. Radiology. 2017 Nov; 285(2):670-80.

26 Shim JH, Jun MJ, Han S, Lee YJ, Lee SG, Kim KM, et al. Prognostic nomograms for prediction of recurrence and survival after curative liver resection for hepatocellular carcinoma. Ann Surg. 2015 May;261(5):939-46.

27 Yang P, Qiu J, Li J, Wu D, Wan X, Lau WY, et al. Nomograms for Pre- and Postoperative Prediction of Long-term Survival for Patients Who Underwent Hepatectomy for Multiple Hepatocellular Carcinomas. Ann Surg. 2016 Apr;263(4):778-86.

28 Kim Y, Park HC, Yoon SM, Kim TH, Lee J, Choi J, et al. Prognostic group stratification and nomogram for predicting overall survival in patients who received radiotherapy for abdominal lymph node metastasis from hepatocellular carcinoma: a multi-institutional retrospective study (KROG 15-02). Oncotarget. 2017 Oct; 8(55):94450-61.

29 Wee CW, Kim K, Chie EK, Yu SJ, Kim YJ, Yoon JH. Prognostic stratification and nomogram for survival prediction in hepatocellular carcinoma patients treated with radiotherapy for lymph node metastasis. Br J Radiol. 2016 Sep;89(1065):20160383. 